\title{
TURBO CODED OFDM SYSTEM WITH PEAK POWER REDUCTION
}

\author{
Mao-Chao Lin and Kuan-Cheng Chen \\ Department of Electrical Engineering and \\ Graduate Institute of Communication \\ Engineering, National Taiwan University, \\ Taipei 106, Taiwan, R.O.C. \\ Tel:+886-2-23635251 ext. 323 \\ Fax:+886-2-23638247 \\ E-mail:mclin@cc.ee.ntu.edu.tw
}

\author{
Sherg-Lung Li \\ Quanta Research Institute, \\ Quanta Computer Inc. \\ No.4, Wen Ming 1 St, Kuei Shan Hsing, \\ Tao Yuan Shien, Taiwan, R.O.C. \\ Tel:0968332801 \\ E-mail:Shoryu.Lee@quantatw.com
}

\begin{abstract}
We propose three turbo coded Orthogonal frequency-division multiplexing (OFDM) systems which can mitigate the peak to average power ratio (PAPR) problem. For the first, we use long maximum-length sequences as test sequences to increase the capability of PAPR reduction and use a short code to protect the side information. Sufficient protection of the side information is obtained by increasing the power of subcarriers corresponding to the short code. For the second, we use several distinct interleavers to yield output symbols with various PAPR and then select the one with the lowest PAPR. In this way, no side information is needed. For the third, a combination of test sequences and interleavers are used. Simulation results show that all the three systems can achieve significant PAPR reduction and satisfactory error performance over AWGN channels.
\end{abstract}

\section{Introduction}

A well known problem of the orthogonal frequencydivision multiplexing (OFDM) [1] system is the possible occurrence of high peak to average power ratio (PAPR). Many techniques have been proposed to mitigate the PAPR problem. Except for the signal distortion techniques $[2,3,4]$ such as clipping, peak windowing and companding, redundancy is needed to control PAPR. The redundancy based PAPR reduction techniques include selective mapping, partial transmit sequence, tone reservation, tone injection and coding, etc. $[5,6,7,8]$. In particular, selective mapping [5] has the flexibility of adjusting PAPR depending on the size of the set of test sequences or equivalently the size of side information. In the applications over noisy channels, the protection of side information is essential. In [9], the original data sequence is scrambled in a way determined by side information and the scrambled sequence is protected by additional redundancy using a coding scheme such as bit interleaved coded modulation (BICM), where the side information appears explicitly in the scrambled sequence. The advantage of the scheme in [9] is that no explicit protection is needed for side information and the rate loss due to the side information is small. However, since the scrambled sequence is limited to the message part of the coded output, there is room for further PAPR reduction. Furthermore, in order to reduce the effect of error propagation resultant from the usage of scrambler, the degree of feedback polynomial must be small and hence the choice of possible scrambled sequences is further restricted. In this paper, we consider three turbo coded OFDM systems PAPR reduction capability. For the first, we propose to use selective mapping technique for which the test sequence is applied to the whole turbo codeword. We choose maximum-length sequences (m-sequences) with both length and period comparable to the length of turbo codeword as test sequences. Such an arrangement is equivalent to applying the turbo codeword through a scrambler which can generate m-sequences with period comparable to the length of turbo codeword under the condition of zero input. In this way, the probability of the occurrence of high PAPR can be significantly reduced. The problem of such an arrangement is that the side information is not protected by the turbo coding and hence additional redundancy is needed for protecting the side information. We find that using simple and short coding with increased subcarrier power to protect the side information will be good enough. The second system with PAPR reduction capability considered in this paper is a turbo coded OFDM system equipped with $Q$ distinct interleavers. For each transmission of information, the PAPR of the turbo coded OFDM symbol resultant from one of the $Q$ interleavers may be different from the PAPR resultant from another inter- 
leaver. The turbo coded OFDM symbol with the lowest PAPR is then transmitted to the receiver. The receiver needs to use turbo decoding for each of the $Q$ distinct interleaver. The interleaver for the decoder that yield the best reliability of the decoded information is considered as the interleaver used in the transmiiter. The advantage of such a PAPR reduction technique is that no side information is needed and there is no rate loss. The disadvantge is the increased decoding complexity. The third system considered in this paper is a turbo coded OFDM system which uses a combination of test sequences and interleavers to achieve PAPR reduction. Tradeoff between the size of side information and the decoding complexity can be achieved. Simulation for an 256 BPSK subcarriers rate $1 / 2$ turbo coded OFDM example with oversampling factor of 4 shows that all the three system can provide both significant PAPR reduction and low bit error rate over the additive white Gaussian noise (AWGN) channel.

\section{System 1 : Turbo Coded OFDM System Using m-sequences}

We present this system by using an example for which the transmitter given in Fig. 1. The 124-bit message sequence $\bar{u}$ is encoded by a rate $1 / 2$ binary turbo code to result in a 248-bit turbo coded sequence $\bar{v}$. The 4 -bit side information $\bar{s}$ is used to select one sequence from a set of 16 randomly chosen m-sequences of period 255 , the first 248 bit of which denoted by $\bar{t}(\bar{s})$ is then added to the turbo coded sequence $\bar{v}$. The side information $\bar{s}$ is encoded by a $(8,4)$ Reed-Muller coded to yield an 8-bit codeword $(\bar{s}, \bar{p})$, where $\bar{p}$ is the 4-bit parity check. The 256 values of $(\bar{v} \oplus \bar{t}(\bar{s}), 1.5 \bar{s}, 1.5 \bar{p})$ are mapped to the 256 subcarriers of the OFDM system. The final output is the one with smallest PAPR out of the 16 possible ouput resultant from 16 possible $\bar{u}$. Note that to increase the reliability of side information, the amplitude of the 8 subcarriers corresponding to $(\bar{s}, \bar{p})$ is increased by a factor of 1.5. The probabilities of occurrence of various PAPR obtained by simulation are shown in Fig. 2. For comparison purpose, we include the other three cases in Fig. 2. For both case A and case $\mathrm{B}$, the 128-bit message sequence added with test sequence, $\bar{u}^{\prime} \oplus \bar{t}^{\prime}(\bar{s})$, is encoded into a 256-bit turbo coded sequence $\bar{v}^{\prime}$ which are then mapped to the 256 subcarriers, where $\bar{t}^{\prime}(\bar{s})$ is an m-sequence of length 128 with period 127 for case A and period 15 for case B respectively. For case $\mathrm{C}$, the 256 code bits of the rate $1 / 2$ turbo coded sequence without any PAPR reduction technique are mapped to the 256 subcarriers. From the simulation results given in Fig. 2, we can see that the system 1 has significantly better PAPR reduction. We also note that restricting the length of test sequence such as case A and case B does limit the capability of PAPR reduction. The effectiveness of PAPR reduction can also be found in Fig. 3, in which the power spec- tra of case $\mathrm{C}$ and the proposed design under $3 \mathrm{~dB}, 6$ $\mathrm{dB}, 7 \mathrm{~dB}$ and $9 \mathrm{~dB}$ clippers respectively are displayed. These results match the observations in Fig. 2, where we see that both the system 1 and case $\mathrm{C}$ will have similar power spectra for the $3 \mathrm{~dB}$ clipper, the system 1 will have better power spectra for the $6 \mathrm{~dB}$ and $7 \mathrm{~dB}$ clippers. Since we use a simple short code to protect the side information, the resultant rate loss is small. Simulation results over AWGN channel given in Fig. 4 show that for $3 \mathrm{~dB}$ and $6 \mathrm{~dB}$, the system 1 has only slightly inferior bit error rate (BER) performance as compared to case $\mathrm{C}$. The inferior performance of the system 1 comes from the rate loss and the increased power for subcarriers corresponding to the short code. The loss of error performance is within the range of $10 \log \left[\frac{(1.5)^{2} \times 8+248}{256}\right]-10 \log \frac{248}{256} \approx 0.3 \mathrm{~dB}$. This phenomenon demonstrates that the increased power for subcarriers corresponding to the short code can provide sufficient protection for the side information.

\section{System 2 : Turbo Coded OFDM System Using Distinct Interleavers}

Consider the transmitter as shown in Fig. 5, where $Q$ distinct interleavers are used. For the $i$-th interleaver, we encode the 128 -bit message sequence $\bar{u}$ by a rate $1 / 2$ binary turbo code to result in a 256-bit turbo coded sequence $\bar{A}_{i}$, which is converted into $\bar{a}_{i}$ through the IFFT operation. Then, $\bar{a}_{j}$ is selected for transmission if $\bar{a}_{j}$ has PAPR no greater than $\bar{a}_{i}$ for $i \neq j$. The structure of the receiver is shown in Fig. 6 , where $\left(\bar{x}_{k}, \bar{y}_{k}\right)$ represents the possibly error-corrupted form of the $k$-th information bit and the $k$-th parity bit, $k=1,2, \cdots, 128$. There are $Q$ decoders corresponding to $Q$ interleavers respectively. The $i$-th decoder computes the reliability

$$
\tilde{L}_{i}^{r}\left(d_{k}\right)=\log \left[\frac{\operatorname{prob}\left\{d_{k}=1 \mid R\right\}}{\operatorname{prob}\left\{d_{k}=0 \mid R\right\}}\right],
$$

for the $k$-th information bit at the $r$-th iteration, where $R=\left\{\left(\bar{x}_{1}, \bar{y}_{1}\right),\left(\bar{x}_{2}, \bar{y}_{2}\right), \cdots,\left(\bar{x}_{128}, \bar{y}_{128}\right)\right\}$. At the $S$-th iteration of decoding, $\beta_{i}=\sum_{k=1}^{128} \tilde{L}_{i}^{S}\left(d_{k}\right)$ is computed for each $i$. The $j$-th decoder is selected if $\beta_{j}$ is no less than any $\beta_{i}$ for $i \neq j$. Only the $j$-th decoder needs to continue its decoding until the $I$-th iteration and yields the hard decision on $\tilde{L}_{j}^{I}$ as the decoded output. Note that the complexity of such a receiver will be $[(N-$ 1) $S+I] / I$ times of the that of case C, i.e., the original turbo coded OFDM system without PAPR reduction design. In this paper, we use interleaver design based on the results in [10]. The index mapping function $\Omega$ : $i \rightarrow j$ is expressed by

$$
j=\Omega(i)=\frac{k}{2}+i+\frac{\sqrt{k^{2}+8 k i}}{2}(\bmod n)
$$

where $n$ is the size of interleaver and $k$ is an odd integer. Let $n=128$. Hence, it is possible to choose 64 distinct 
$k$ values which corresponding to 64 interleavers. In the simulation, we consider $Q=16$ and choose $k=$ $\{35,37,39,41,45,49,57,59,69,71,81,87,89,103,107$, $121\}$. From Fig. 2, we observe that a $2.5 \mathrm{~dB}$ PAPR reduction can be achieved for clipping probability below 0.0001 as compared to case C. The associated power spectral density is given in Fig. 7. Compared to case $\mathrm{C}$, more than $1 \mathrm{~dB}$ can be saved in the backoff for $-40 \mathrm{~dB}$ out-of-band level in addition to the 0.5 $\mathrm{dB}$ improvement of BER at the level of $10^{-5}$. The price to pay is that the decoding complexity is 8.5 times as great. We note that the PAPR reduction of system 2 is not as significant as that of system 1 although system 2 has better BER performance. The inferior performance of PAPR reduction of system 2 as compared to system 1 may be due to the design that different interleavers will only cause variation of the 64 parity bits resultant from systematic encoder 2 of the turbo code. In case that highly PAPR reduction and low decoding complexity are needed, we suggest a combination of various test sequences and various interleavers be used, which will be the system described in section IV.

\section{System 3 : Turbo Coded OFDM System Using Distinct m-sequences and Distinct Interleavers}

This system is almost a combination of case A described in section II and system 3 described in section III except that 2 message bits in the 128-bit sequence $\bar{u}^{\prime}$ must be replaced by 2 side-information bits and the number of distinct interleavers are set to be 4 . In this way, in the transmitter, for each input of a 126-bit information sequence, there are 16 distinct outputs of 256subcarriers OFDM symbol. The one with the lowest PAPR is sent for transmission. The decoding complexity of this sytem is 2.5 times of that of case C. In the simulation, the $k$ parameters for the 4 interleavers are 59, 87, 89 and 103 respectively. From Fig. 2, 3 and 8 , we can see that the PAPR reduction capability of system 3 is only slightly inferior to that of system 1 . However, system 3 has better BER performance and higher transmission rate.

\section{Conclusion}

We have proposed three turbo coded OFDM systems with PAPR reduction capability. The presentation is this paper is given based on the subcarriers with BPSK modulations. However, the idea can be easily adapted to subcarriers with other modulations such as QPSK and16QAM.

\section{References}

[1] R. Van Nee, "COFDM for Wireless Multimedia Communication," Artech House, 2000.
[2] Xiaodong Li, Cimini, L.J., Jr., " Effects of clipping and filtering on the performance of OFDM," Vehicular Technology Conference, vol. 3, pp. 1634-1638, May 1997.

[3] van Nee, R., de Wild, A.," Reducing the peak-toaverage power ratio of OFDM," Vehicular Technology Conference, vol. 3, pp. 2072-2076, May 1998.

[4] Xianbin Wang, Tjhung, T.T., Ng, C.S., " Reduction of peak-to-average power ratio of OFDM system using a companding technique," Broadcasting, IEEE Transactions on, vol. 45, no. 3, pp. 303-307, September 1999.

[5] R. Bauml, R. Fisher, J. Huber, "Reducing The Peakto-Average Power ratio of Multicarrier Modulation by Selected Mapping," Electronics Letters, vol. 32, no. 22, pp. 2056-2057, October 1996.

[6] Muller, Huber, J.B., " OFDM with reduced peak-toaverage power ratio by optimum combination of partial transmit sequences," Electronics Letters, vol. 33, no. 5, pp. 368-369, February 1997.

[7] J. Tellado, " Multicarrier modulation with low PAPR applications to DSL and wirless," Kluwer academic publishers, 2000.

[8] Wilkinson, T.A., Jones, A.E., " Minimisation of the peak to mean envelope power ratio of multicarrier transmission schemes by block coding," Vehicular Technology Conference, vol. 2, pp. 825-829, July 1995.

[9] M. Breiling, S.H. Muller-Weinfurtner, J.B. Huber, "SLM Peak-Power Reduction Without Explicit Side Information," IEEE Communication Letters, vol. 5, no. 6, pp. 239-241, June 2001.

[10] Takeshita, O.Y., Costello, D.J., Jr., "New deterministic interleaver designs for turbo codes," Information Theory, IEEE Transactions on, vol. 46, no. 6, pp. 19882006, September 2000. 


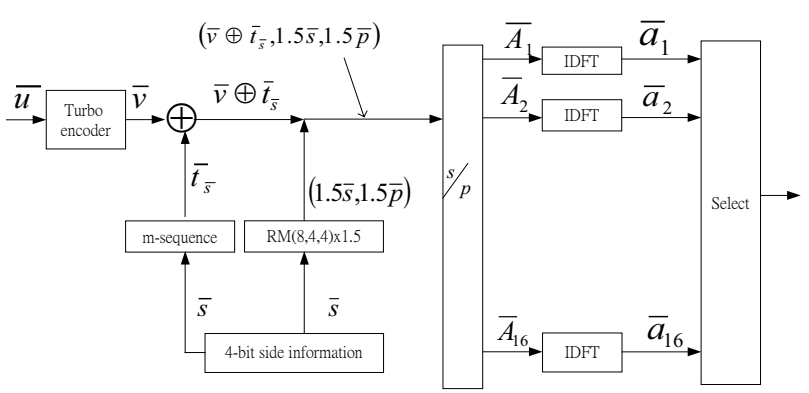

Figure 1: Block diagram of system 1.

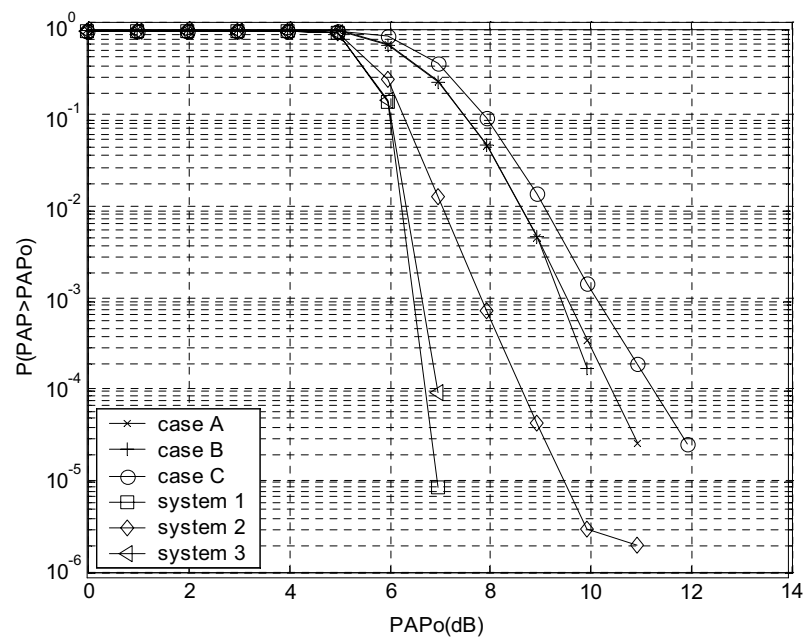

Figure 2: Complementary cumulative distribution of PAPR for several OFDM systems.

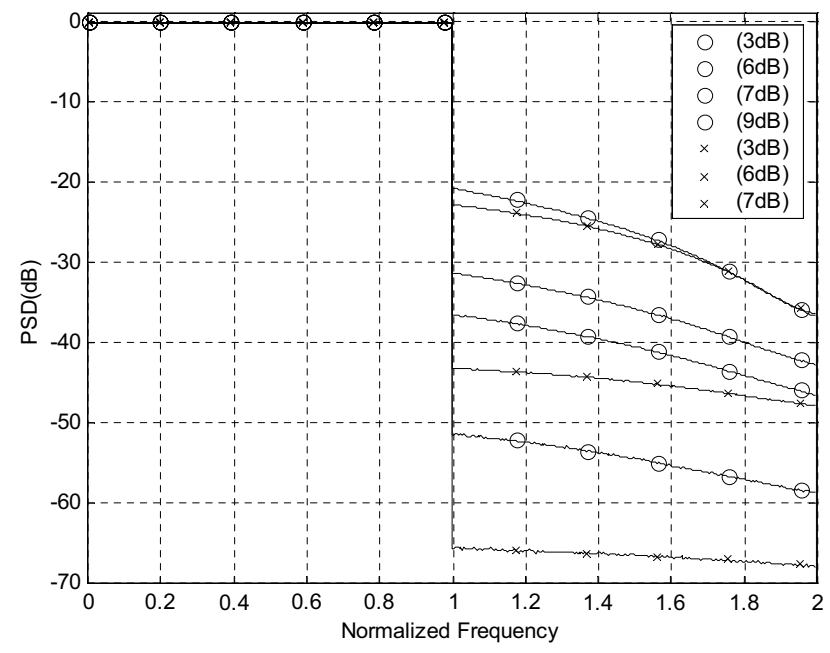

Figure 3: Power Spectra of case C (represented by o) and system 1 (represented by $\mathrm{x}$ ) using various clippers.

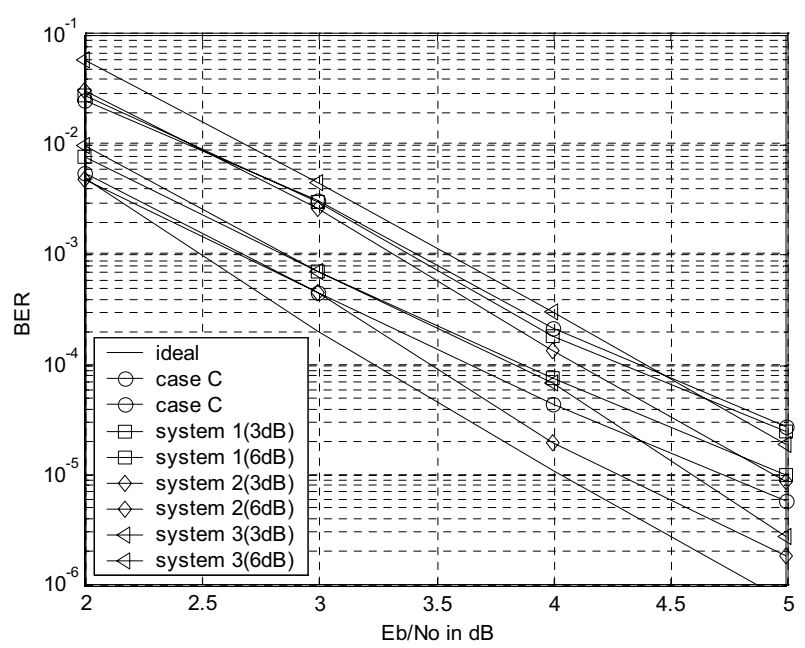

Figure 4: BER of OFDM systems using various clippers.

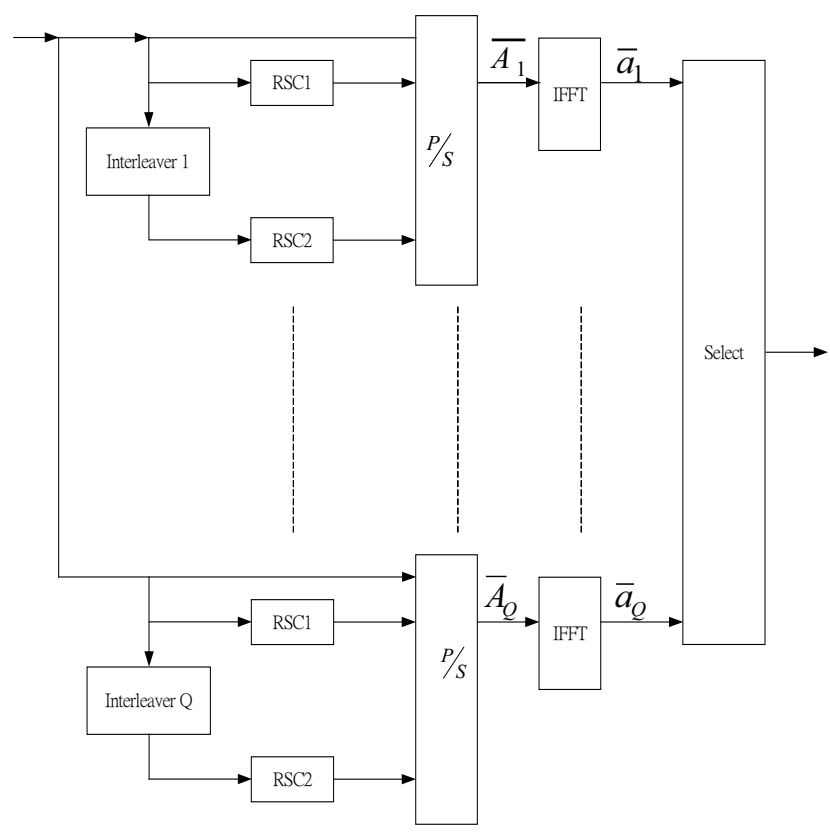

Figure 5: Block diagram of the transmitter of system 2 . 


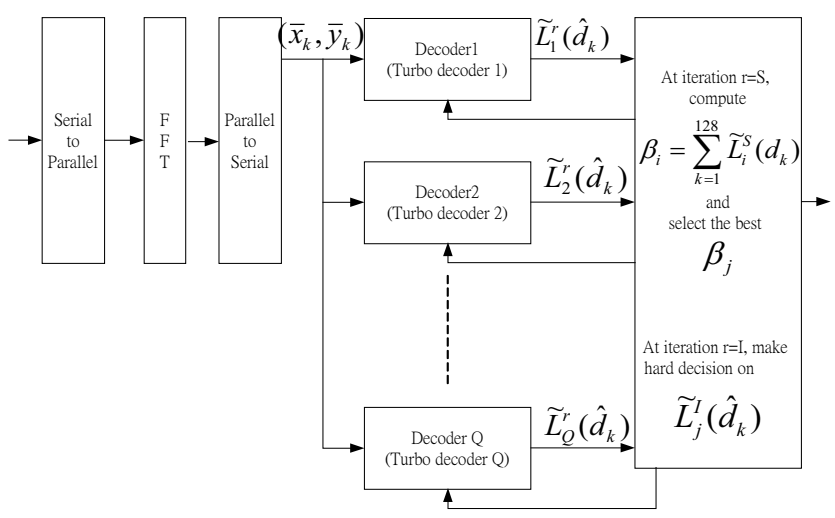

Figure 6: The decoder for system 2.

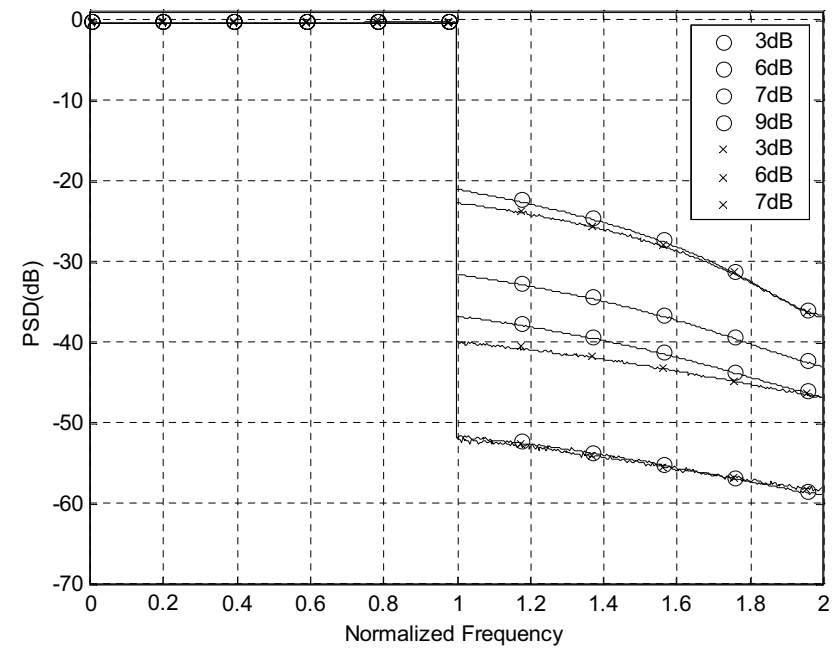

Figure 7: Power Spectra of case C (represented by o) and system 2 (represented by $\mathrm{x}$ ) using various clippers.

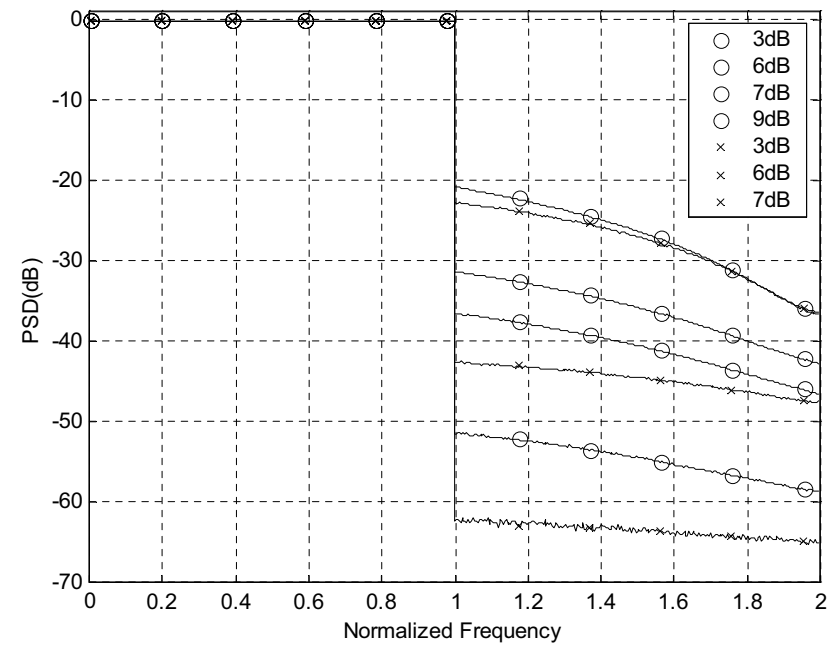

Figure 8: Power Spectra of case C (represented by o) and system 3 (represented by $\mathrm{x}$ ) using various clippers. 